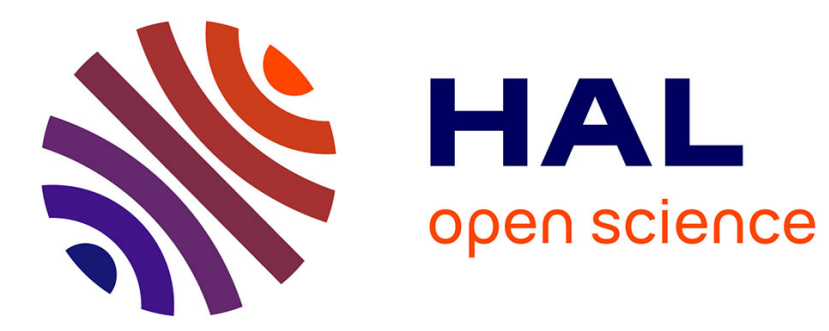

\title{
La gouvernance différenciée des contrats locaux de santé
} Nadine Haschar-Noé, Émilie Salaméro, Marina Honta

\section{To cite this version:}

Nadine Haschar-Noé, Émilie Salaméro, Marina Honta. La gouvernance différenciée des contrats locaux de santé. Journal de gestion et d'économie médicales, 2015, La gouvernance auscultée en santé (1re partie), 33 (6), pp.375-388. 10.3917/jgem.156.0375 . halshs-01367495

\section{HAL Id: halshs-01367495 \\ https://shs.hal.science/halshs-01367495}

Submitted on 16 Sep 2016

HAL is a multi-disciplinary open access archive for the deposit and dissemination of scientific research documents, whether they are published or not. The documents may come from teaching and research institutions in France or abroad, or from public or private research centers.
L'archive ouverte pluridisciplinaire HAL, est destinée au dépôt et à la diffusion de documents scientifiques de niveau recherche, publiés ou non, émanant des établissements d'enseignement et de recherche français ou étrangers, des laboratoires publics ou privés. 


\section{LA GOUVERNANCE DIFFÉRENCIÉE DES CONTRATS LOCAUX DE SANTÉ}

Nadine Haschar-Noé, Emilie Salamero, Marina Honta

ESKA | « Journal de gestion et d'économie médicales »

2015/6 Vol. 33 | pages 375 à 388

ISSN 2262-5305

ISBN 9782747225946

Article disponible en ligne à l'adresse :

http://www.cairn.info/revue-journal-de-gestion-et-d-economiemedicales-2015-6-page-375.htm

\section{Pour citer cet article :}

Nadine Haschar-Noé et al., « La gouvernance différenciée des contrats locaux de santé », Journal de gestion et d'économie médicales 2015/6 (Vol. 33), p. 375-388.

Distribution électronique Cairn.info pour ESKA.

(C) ESKA. Tous droits réservés pour tous pays.

La reproduction ou représentation de cet article, notamment par photocopie, n'est autorisée que dans les limites des conditions générales d'utilisation du site ou, le cas échéant, des conditions générales de la licence souscrite par votre établissement. Toute autre reproduction ou représentation, en tout ou partie, sous quelque forme et de quelque manière que ce soit, est interdite sauf accord préalable et écrit de l'éditeur, en dehors des cas prévus par la législation en vigueur en France. Il est précisé que son stockage dans une base de données est également interdit. 


\title{
La gouvernance différenciée des contrats locaux de santé
}

\section{The Differentiated Governance of Local Health Contracts}

\author{
Nadine Haschar-Nó́*, Emilie SAlaméro** et Marina Honta*** \\ * Maître de conférences, Laboratoire creSco (EA 4561), \\ Institut d'études et de recherches interdisciplinaires Santé-société (Ifériss), \\ Université de Toulouse, France. \\ F2SMH, Université Paul Sabatier, 118, route de Narbonne, \\ 31062, Toulouse cedex 09. \\ ** Maître de conférences, Laboratoire CEREGE (EA 1722), \\ Université de Poitiers, France. \\ Faculté des Sciences du sport, Bâtiment C6, 8 allée Jean Monnet, \\ 86073 Poitiers cedex 9.
}

*** Professeur des universités, Centre Emile Durkheim (UMR CNRS 5116), Université de Bordeaux, France. Université de Bordeaux, 3 ter place de la Victoire, 33076 Bordeaux cedex.

\author{
Auteur de correspondance: \\ Nadine Haschar-Noé \\ hascharnoe@orange.fr \\ Le Crouzet, 81470 Montgey \\ $0563757492 / 0682022800$
}

\section{RÉSUMÉ}

Selon une sociologie politique considérant l'action publique comme une construction collective d'acteurs publics et privés en interaction, notre recherche se focalise sur la «nouvelle» gouvernance sanitaire des «territoires de santé » promue par les Agences régionales de santé (ARS) en s'intéressant aux modalités de fabrication des contrats locaux de santé (CLS). Dans la région étudiée, l'ARS lance, dès octobre 2010, les deux premiers CLS comme une forme 
d'expérimentation in vivo, avant leur future généralisation. A partir d'une analyse comparée des sept CLS signés en 2014, nos résultats montrent, d'une part, que leur gouvernance négociée donne lieu à d'importantes variations territoriales bien que domine une approche populationnelle légitimant l'action des élus locaux. D'autre part, via une enquête socio ethnographique de deux CLS en train de se faire, nous montrons que leur mode de négociation par l'ARS oscille entre deux logiques de gouvernance, descendante ou ascendante, «dure ou molle», et qu'un certain nombre de conditions favorables participent à la construction des accords et des compromis acceptables pour les diverses parties prenantes. Loin de prendre les formes d'une gouvernance «unifiée», fabriquer un CLS s'apparente davantage à faire du «sur mesure». Les acteurs qui s'y engagent sont ainsi condamnés à s'entendre pour répondre, selon un jeu d'instrumentalisations réciproques, aussi bien aux problématiques «spécifiques» des territoires infrarégionaux qu'aux objectifs du Plan régional de santé.

Mots-clés : instrument d'action publique, territorialisation, instrumentalisation réciproque.

\begin{abstract}
According to a political sociology considering public action as a collective construction of interacting public and private players, our research focuses on the "new" governance of the "health territories" promoted by the Regional Health Authorities (ARS), and looks into the ways in which Local Health Contracts (CLS) have been produced. In the study area, the ARS launched its first two CLS in October 2010, as a sort of live experiment before rolling them out more generally in the future. The results of a comparative analysis of the seven CLS signed in 2014 show first that their negotiated governance gives rise to significant territorial variations, although a populational approach does dominate, legitimising the action of local elected office holders. Next, through a socio-ethnographic survey of two CLS in their formation process, we show that the way they are negotiated by the ARS oscillates between two different approaches to governance, top-down or bottom up, "hard or soft", and that there are a certain number of favourable conditions that contribute to the construction of agreements and compromises that are acceptable to the various stakeholders. Far from taking the form of one "unified" governance, the production of CLS tends to be more "customised". The stakeholders that take part therefore have no choice but to come to an agreement in order to respond, through a process of mutual instrumentalisation, not only to the specific issues of the sub-regional territories, but also to the objectives of the Regional Health Plan.
\end{abstract}

Keywords: public action instrument, territorialisation, mutual instrumentalisation. 
En France, le gouvernement local subit des transformations majeures depuis une trentaine d'années : fragmentation, retrait de l'Etat, accroissement du rôle du secteur privé. Ces évolutions ont donné lieu à des tentatives pour conceptualiser cette nouvelle forme de gouvernement avec le terme d'origine anglo-saxonne de gouvernance. Rendant problématique l'intervention de l'Etat, ces recompositions conduisent à s'interroger sur les conditions d'existence des stratégies locales, l'éventuelle formalisation de régulations sociales et politiques au sens de règles du jeu stabilisées entre acteurs et enfin la façon dont les politiques nationales sont intégrées, traduites, transformées ou rejetées dans ces stratégies [1].

Dans ce contexte et selon une sociologie politique analysant l'action publique comme une construction collective d'acteurs publics et privés en interaction, notre recherche se focalise sur la «nouvelle» gouvernance sanitaire des «territoires de santé» [2] promue par les Agences régionales de santé (ARS) en s'intéressant aux modalités de fabrication des contrats locaux de santé (CLS). Dans la région étudiée, dès octobre 2010, l'ARS lance ses deux premiers CLS comme une forme d'expérimentation in vivo, avant leur future généralisation. Cette démarche de contractualisation se traduit en 2013 et 2014 par la signature de sept CLS qui constituent les terrains empiriques de la recherche présentée.

Les CLS se situent dans la continuité des Contrats locaux de santé publique annoncés dans le Plan espoir banlieues en 2008: prolongements contractuels des Ateliers santé ville (ASV), ils sont intégrés au Plan régional de santé publique. La circulaire interministérielle du 20 janvier $2009^{1}$ annonce la mise en place de CLS visant à soutenir et amplifier les initiatives en santé au sein des Contrats urbains de cohésion sociale. Ces éléments sont confirmés par la loi Hôpital patients santé et territoires (HPST) du 21 juillet 2009 qui prévoit que la mise en œuvre du Projet régional de santé (PRS) peut faire l'objet de CLS conclus par «l'agence, notamment avec les collectivités territoriales et leurs groupements, portant sur la promotion de la santé, la prévention, les politiques de soins et l'accompagnement médico-social $\gg^{2}$. En 2011 , une note remise aux ministères concernés et un «kit méthodologique $»^{3}$ destiné aux ARS visent à fixer un cadre national de référence et un pilotage «unifié» de ces contrats préconisant leur articulation avec le PRS, l'élargissement des actions locales pour les intégrer dans les CLS et les faire correspondre aux compétences de l'ARS. Cependant, le caractère «flou» et peu cadré a priori de ce dispositif, propre par ailleurs aux politiques processuelles et constitutives [3], laisse une importante marge de manœuvre aux acteurs qui s'y engagent et soulève par conséquent des enjeux de gouvernance autrement dit: «de coordination d'acteurs, de groupes sociaux, d'institutions pour atteindre des buts propres discutés et définis collectivement dans des environnements fragmentés, incertains » [1]. Peu étudiés à ce jour, les CLS constituent un terrain heuristique pour saisir les formes que prend la gouvernance territoriale saisie comme une reconfiguration d'interdépendances entre acteurs engagés dans l'action publique sanitaire et autorisant un jeu plus ouvert, plus diffus et plus complexe mais aussi très différencié selon les configurations locales ou sectorielles [4].

L'approche conceptuelle choisie considère le CLS comme un instrument d'action publique pouvant faire l'objet d'espaces et de formes de résistances différenciés [5]. Il constitue aussi

\footnotetext{
DGS/DIV/DP1 n² 2009-68 (NOR : SASP09301197Y).

Art. L. 1434-17 du Code de la Santé Publique

http://www.ars.iledefrance.sante.fr/Le-kit-methodologique.116167.0.html
} 
un contrat politique d'action publique soit «des procédures d'accord formalisé, portant sur des objectifs à moyen terme et qui impliquent, pour leur réalisation, des contributions conjointes » [6]. Ainsi, en tant qu'instrument de gouvernance territoriale, il nécessite la fabrication de compromis [7] entre de multiples acteurs censés favoriser la territorialisation des plans nationaux et régionaux et la mise en cohérence des politiques sanitaires locales sous pilotage régional [8].

A partir d'une analyse comparée de CLS signés dans une même région, nous montrerons que leur gouvernance donne lieu à de fortes différenciations tant elle est traversée par une tension entre «le dur et le mou, le solide et le labile» $[4,9]$ qui fluctue selon les contextes et configurations d'acteurs en présence. Relativement autonome [10], la gouvernance de ces contrats par l'ARS oscille entre: une logique descendante dans laquelle l'ARS, selon un modèle de type «préfectoral» [11], tente d'imposer son leadership territorial [12] auquel résistent les acteurs locaux; une logique ascendante plus participative, favorisée par ce «nouvel» instrument contractuel dont se saisissent des systèmes d'acteurs territorialisés pour ajuster le CLS à leur territoire d'action et d'élection et affirmer ainsi leur légitimité d'agir en santé. Ainsi, la gouvernance d'un CLS s'apparente davantage à fabriquer du «sur mesure», condamnant les acteurs qui s'y engagent à s'entendre pour répondre à la fois aux problématiques «spécifiques » des territoires infrarégionaux et aux objectifs du PRS, selon un jeu d'instrumentalisations réciproques dans lequel chaque niveau appréhende l'autre comme une structure d'opportunité afin de dépasser les blocages qui limitent son action [13].
Nos résultats s'appuient sur une analyse menée à deux niveaux. Le premier consiste à dégager les principaux analyseurs de leur gouvernance différenciée (1). Le second niveau, au grain d'analyse plus fin, s'appuie sur une socio-ethnographie de deux CLS «en train de se faire» [14] choisis pour leur «exemplarité» contrastée, tant en termes de contextes que de jeux d'acteurs et de modes de gouvernance (2).

\section{Encart $\mathbf{n}^{\circ} 1$ : Méthodologie}

Menée d'octobre 2010 à juillet 2014, notre recherche combine plusieurs méthodologies de recueil de données selon une double visée scientifique et pragmatique ${ }^{4}$. Nous avons ainsi réalisé:

- une analyse de contenu des documents de travail produits par les instances régionales et locales sur leur politique de santé et CLS, complétée par une revue de la presse locale;

- des observations lors des réunions de tous les comités (de pilotage, technique et de rédaction) de deux CLS (de leur lancement à leur signature) et la tenue d'un carnet de terrain ethnographique;

- la réalisation de 29 entretiens semi-directifs auprès de l'ARS et de ses délégations territoriales (DT) $(n=4)$, des acteurs infrarégionaux ayant participé aux divers comités des trois premiers CLS signés ( $\mathrm{n}$ $=25$ ): élus, réseaux de santé, organismes d'assurance maladie, services de l'Etat territorial, associations et entreprises, représentants d'usagers, de médecins libéraux et hospitaliers, techniciens territoriaux, etc.

\footnotetext{
4 Grâce au partenariat avec l'ARS nous avons pu mener une recherche socio ethnographique. Etant invitées comme observatrices aux réunions des comités et accédant à leurs documents internes de travail, nous étions au cœur des processus que nous souhaitions étudier. En retour, un certain nombre de préconisations ont été proposées à l'ARS sur les conditions susceptibles de favoriser une construction négociée des CLS.
} 


\section{LES DIFFÉRENCIATIONS TERRITORIALES DES CLS}

Selon les données disponibles, la région étudiée se situe en position médiane dans la dynamique de signature des CLS. En effet, au 31 décembre 2013, 202 CLS ont été signés dans 27 régions françaises mais selon un rythme et une densité très contrastés. Si $28 \%$ des CLS signés l'ont été en Ile de France, les autres se répartissent selon des dynamiques régionales hétérogènes: dix signatures et plus pour quatre régions ; moins de cinq signatures pour 12 régions; et entre cinq et dix signatures pour dix régions dont celle étudiée. $\mathrm{La}$ comparaison des sept CLS signés dans cette région permet, à partir de la construction de deux types d'analyseurs transversaux, de repérer et d'expliquer les différenciations territoriales observées. Elle confirme qu'en dépit de la fragmentation institutionnelle des scènes décisionnelles en région et de la recomposition des relations entre l'Etat et les collectivités territoriales suite aux différents «Actes» de décentralisation, des négociations propices à la production de projets collectifs peuvent agréger les diverses parties prenantes [15].

\subsection{Temporalités et "pertinence» des territoires: les «bassins de santé» en question}

La comparaison des dynamiques temporelles, spatiales et partenariales ayant conduit à la signature des CLS s'appuie sur plusieurs indicateurs qui illustrent les variations dont ces contrats font l'objet: tant sur les délais de leur fabrication et leur durée que sur la définition de leur périmètre et territoire d'action «pertinent» [16] et ses conséquences sur le volume des populations concernées, les nombre et type de signataires et de «partenaires» impliqués et la plus ou moins grande complexité à construire des accords.

Leur délai de fabrication s'étale de 15 à 40 mois selon une temporalité continue pour certains ou nettement plus heurtée et marquée par des conflits et l'arrêt des négociations pour d'autres. Leur durée est elle aussi relativement variable allant de quatre à deux ans. Ces temporalités hétérogènes sont en relation avec le choix plus ou moins conflictuel du périmètre «pertinent» du CLS. En effet, sa définition «idéale» initialement prévue par l'ARS correspond aux «bassins de santé », découpage réalisé par l'agence et basé sur l'offre de soins de premier recours. Or, le choix de ce périmètre est éminemment stratégique [17] car il est à la fois un espace géographique, un regroupement d'acteurs et une arène politique. Il a donc parfois fait l'objet de négociations avec les élus locaux, soucieux de faire coïncider le périmètre proposé par l'ARS avec celui de leurs territoires d'action et d'élection. Ainsi, si le périmètre apparaît comme un indicateur «technique» ou simplement "géographique» pour l'ARS, sa définition revêt une forte dimension politique car il définit aussi un territoire d'action à construire comme bien commun. Ainsi, périmètre et territoire du CLS peuvent se heurter selon leur superposition, partielle ou totale, avec les découpages électoraux, les «bassins de vie» vécus par les populations et les politiques sanitaires «déjà là» des collectivités territoriales. Par exemple, la définition du territoire d'action du premier CLS signé a fait l'objet de conflits récurrents avec le conseil départemental dont la politique médico-sociale est structurée de longue date selon un découpage en quatre Pays et non en «bassins de santé». Appliqués sur ce territoire à (ré)affirmer leurs compétences en matière d'action sociale et médicosociale et leur relative autonomie financière et politique $[10,18]$, les élus départementaux ont négocié «pied à pied» 
la création d'une seule Maison pour l'autonomie et l'intégration des malades d'Alzheimer (MAIA) au lieu des quatre prévues par l'ARS. Cette condition «non négociable» pour qu'ils signent le CLS, visait à faire «... respecter le schéma général autonomie voté démocratiquement par l'assemblée départementale et consolider le conseil général comme le chef de file du médico-social en l'adossant à la Maison départementale des personnes handicapées $\gg^{5}$. Ainsi, la grande variété des périmètres des sept CLS signés apparaît comme la résultante de conflits et de choix, plus ou moins longuement et âprement négociés entre l'ARS et les collectivités territoriales signataires. In fine, un seul périmètre correspond à celui d'un «bassin de santé » de l'ARS, les six autres étant communaux (2), intercommunaux (2), cantonaux et/ou de Pays (2).

En outre, si les périmètres des CLS signés recouvrent une population totale de 626506 habitants soit $21,5 \%$ de la population régionale, ils touchent un volume très variable allant de 20500 à 440204 habitants, soit une différence de un à vingt. Cette définition du périmètre et du «territoire» du CLS a aussi des conséquences majeures sur le nombre et le type de signataires à mobiliser. Par là même, s'accroît la complexité de construire des accords et de négocier des compromis [7] entre de multiples acteurs publics et privés situés dans des secteurs et niveaux d'action différents. Si la variabilité numérique des signataires est importante (de deux à dix pour une moyenne de 4,8), leurs types sont relativement homogènes. Pour les sept CLS étudiés, trois catégories dominent mais selon une fréquence variable donnant à voir des configurations d'acteurs changeantes car soumises à négociations :

- des représentants de l'Etat territorial (7/7 ARS, 2/7 préfecture, 2/7 DDSEN) ${ }^{6}$;

- des organismes d'assurance maladie et de prestations sociales (3/7 CPAM, 2/7 MSA et 2/7 CAF);

- des collectivités ou leurs groupements (2/7 Conseil départemental, 2/7 Pays, 2/7 EPCI et 4/7 communes) et, très rarement, des centres hospitaliers (1/7).

Assortis le plus souvent d'un nombre important de «partenaires » ${ }^{7}$, les CLS apparaissent dans tous les cas comme des instruments de mobilisation et d'enrôlement des acteurs locaux parmi lesquels figurent par ordre d'importance: des directions départementales ou régionales autres que sanitaires, des établissements hospitaliers, médicaux, médicosociaux ou sociaux, des collectivités non signataires mais situées dans le périmètre du CLS, des professionnels de santé libéraux ou hospitaliers, des associations de prévention/ promotion de la santé, des réseaux de santé et, plus rarement, des entreprises.

\subsection{Des CLS polarisés sur une approche populationnelle}

Un deuxième type d'analyseur a permis de repérer, dans chacun des contrats signés, les axes d'intervention choisis comme prioritaires et les fiches-actions retenues pour leur mise en œuvre.

\footnotetext{
Tous les passages entre guillemets et en italique sont des extraits d'entretiens ou du carnet de terrain ethnographique.

' Lecture : l'ARS est signataire de 7 CLS sur les 7 signés, la préfecture de 2 CLS sur 7 tout comme la Direction départementale des services de l'éducation nationale (DDSEN).

7 Acteurs associés aux programmes d'action du CLS mais qui ne les pilotent ou ne les financent pas et ne sont pas signataires.
} 


\section{Encart $\mathbf{n}^{\circ} 2$ : La construction des catégories d’analyse}

A partir de nos données de terrain, nous avons construit cinq catégories d'analyse autorisant une comparaison des axes et fiches-actions des CLS. Trois catégories correspondent aux champs d'intervention traditionnels de l'ARS et se polarisent entre approche thématique et populationnelle. Une première catégorie recouvre «prévention/promotion de la santé»; une deuxième, l' «accès aux soins ou aux droits»; la troisième «mixte» combine approches populationnelle (organisée par âges de la vie ou situations particulières) et thématique (prévention, accès aux soins, aux droits etc.). Enfin deux autres catégories complètent ces trois premières: «l'observation et la coordination » des professionnels et établissements locaux de santé, faisant du CLS un ensemblier et l'instrument d'une stratégie coordonnée; des «partenariats spécifiques » entre l'ARS et le(s) signataire(s) sur des thèmes transversaux comme les déterminants sociaux et environnementaux de santé ou les impacts sanitaires des politiques publiques relevant des compétences des collectivités (transports, emploi, logement, qualité de l'air, programmes éducatifs, sportifs, aménagement de l'espace, etc.).

Si le nombre d'axes prioritaires par CLS est relativement proche (de deux à quatre), la majorité d'entre eux (soit 12/23) sont «mixtes» et à dominante populationnelle: les cibles priorisées sont les personnes âgées, les jeunes et les populations «vulnérables» et, plus rarement, celles désignées par des situations particulières (femmes enceintes, handicapées etc.) ou des pathologies spécifiques (santé mentale, cancer, diabète etc.). Les axes thématiques privilégiant la «prévention/promotion de la santé » et l' «accès aux soins ou aux droits » ne concernent respectivement que trois et deux axes sur les 23 que totalisent les CLS signés. Cette focalisation sur les spécificités sociodémographiques locales est renforcée par six autres axes: trois portent sur des «partenariats spécifiques et/ou des politiques publiques locales » visant à agir sur les déterminants environnementaux ou sociaux de santé et trois autres sur l'amélioration des coordinations entre professionnels et établissements locaux de santé.

La catégorisation des 106 fiches-actions des sept CLS (chacun d'eux proposant de 8 à 25 fiches-actions), permet un grain plus fin d'analyse. Précisons toutefois que le nombre de fiches n'est pas en soi un indicateur de leur opérationnalité directe. En effet, leur construction révèle des niveaux d'opérationnalité et de précision très variables selon les CLS : soit il s'agit d'une fiche décrivant précisément une action, son déroulement, ses porteurs, partenaires et financeurs, ses indicateurs d'évaluation etc.; soit d'une fiche renommée «fiche-orientation» par les acteurs, qui regroupe plusieurs actions poursuivant un même objectif général. Malgré ces nuances, la catégorie des fiches-actions «mixtes», associant une population ciblée et une thématique, est là aussi dominante avec $63 \%$ du total. Les autres catégories se distribuent entre $11 \%$ (accès aux soins et aux droits, observation-coordination), $9 \%$ (prévention/promotion de la santé) et $6 \%$ (partenariats spécifiques et/ ou politiques publiques locales).

Ainsi, la distribution négociée des axes prioritaires et la dimension programmatique des CLS consolident leur dimension territorialisée et leur ancrage dans des préoccupations avant tout sociales puis sanitaires selon une visée de réduction des inégalités. Cette tendance à privilégier les approches populationnelles, apparaît à l'opposé de la 
polarisation sur les thématiques sanitaires des CLS relevée lors d'enquêtes antérieures. En contexte de décentralisation se joue une forme de (re)politisation de la santé par les élus locaux qui trouvent, à l'occasion de la négociation d'un CLS avec l'ARS, une opportunité pour mener des politiques offensives [19]. Elles leur permettent d'affirmer ou de consolider leur légitimité à intervenir en santé [20] malgré la «modestie» de leurs compétences légales dans ce secteur en usant d'arguments combinant proximité d'action, ajustement aux spécificités locales, besoins des populations et attentes de leurs électeurs.

\section{DES GOUVERNANCES D'ACCORDS NÉGOCIÉS À L'ÉPREUVE DES COMPROMIS}

La Loi HPST ne donnant aucune indication sur la gouvernance des CLS, c'est donc aux parties prenantes qu'il appartient d'en définir localement les conditions d'élaboration, les contenus et modalités de mise en œuvre. L'analyse socio-ethnographique de la construction de cette procédure contractuelle entre l'ARS et ses potentiels «partenaires» locaux permet de dégager les conditions favorables et les freins qui président à la signature des CLS et à un gouvernement des compromis acceptables pour les parties prenantes. Le travail ethnographique dévoile les dynamiques sociales très différenciées qui rythment in itinere, les négociations de deux CLS «exemplaires »: pour l'ARS, le premier cas (A) s'apparente à un «cauchemar» et le second $(\mathrm{B})$, à une «belle réussite».

\section{Encart $n^{\circ} 3$ : Focus sur deux CLS contrastés}

Le cas A., situé en milieu rural, est éloigné de la métropole régionale. Il couvre un périmètre de 56 communes, trois intercommunalités et deux cantons rassemblés dans un Pays pour un total de 26943 habitants (Insee, 2011). La mise en place d'un CLS est justifiée par le double constat d'une situation épidémiologique et socio-économique relativement défavorable, d'un taux important de personnes âgées et pour certaines isolées, de problèmes de démographie médicale mais surtout d'une restructuration conflictuelle de l'offre hospitalière et d'indicateurs de précarité sociale alarmants suite à une forte déprise industrielle du territoire.

Le cas B. correspond à une commune périurbaine, proche de la capitale régionale et dynamique sur le plan socio-économique. La population de 35584 habitants (Insee, 2011) est d'un niveau socioéconomique relativement élevé, les indicateurs de santé sont pour la plupart meilleurs que ceux du département et de la région et l'offre sanitaire et médico-sociale est jugée satisfaisante. Cependant, de fortes inégalités de santé et des indicateurs de précarisation persistent dans plusieurs quartiers de la commune qui compte $30 \%$ de logements sociaux qui n'ont jamais été intégrés dans les dispositifs de la politique de la Ville. 


\subsection{Cas A. Pléthore d'acteurs et négociations sous hautes tensions pour un CLS «expérimental» : une gouvernance introuvable}

Entre le lancement du CLS et sa signature, s'écoulent 33 mois (octobre 2010 - juillet 2013) alors que l'ARS prévoyait initialement une durée de sept à dix mois. Cette fabrication ponctuée de conflits entre ARS, collectivités territoriales et acteurs locaux et de périodes d'arrêt des négociations, subit d'importants retards par rapport au calendrier prévu. La démarche contractuelle débute en 2010 dans un contexte d'installation de l'ARS : le Plan stratégique régional de santé (PSRS) et les schémas régionaux en cours d'élaboration laissent les acteurs locaux dans une grande incertitude quant aux orientations sanitaires régionales à venir.

Pour l'ARS, les difficultés de ce CLS «expérimental... qui en paye un peu les pots cassés », tiennent au manque d'expérience et d'habitude de négociation au sein d'une «jeune agence» peu coutumière d'une démarche de projet «réellement concertée » et maitrisant partiellement ce type d'ingénierie. En fait, au-delà de ces arguments, la façon dont ce CLS est lancé «à marche forcée » et piloté par une direction stratégique de l'ARS omniprésente constitue le principal frein à l'instauration d'un dialogue social territorial [21]. De plus, le diagnostic sanitaire du territoire réalisé par
l'Observatoire régional de santé et l'ARS à partir d'indicateurs quantitatifs disponibles mais incomplets faute d'avoir sollicité des opérateurs comme le conseil départemental ou les organismes sociaux, ne convainc pas totalement les élus et professionnels de santé locaux lors de sa présentation à la conférence de territoire. Au cours des premières réunions de travail, la pertinence de ce diagnostic donne lieu à de longs «débats et négociations» sur les aspects qualitatifs devant le compléter et l'affiner, ce qui retarde le choix des axes d'action à privilégier faute d'un accord entre participants.

Enfin, du fait du périmètre géographique étendu voulu par l'ARS ${ }^{8}$, le CLS nécessite la construction d'accords entre un nombre important de signataires ${ }^{9}$ aux intérêts différents, parfois en concurrence et sans habitude de coordination intersectorielle et/ ou interinstitutionnelle [8]. Le tout se double d'une «méfiance réciproque» entre «agence de l'Etat» et élus locaux. Ces derniers refusent d'être «un terrain d'application du PSRS... et de se plier au diktat de l'ARS» tout en tenant des propos ambigus entre nécessité de garantir leur autonomie décisionnelle et de respecter l' «égalité républicaine» que l'Etat se devrait d'assurer [20] pour réduire les inégalités territoriales de santé. Ces tensions se cristallisent autour du maintien du plateau chirurgical de l'hôpital local menacé de fermeture, depuis

\footnotetext{
8 Initialement centré sur un seul canton aux indicateurs sanitaires et sociaux les plus défavorables, le CLS est étendu à la demande de l'ARS, à un second canton voisin. Son directeur fait de la signature du conseil départemental un impératif malgré les réticences des agents de sa DT trouvant la négociation plus complexe «sur un territoire trop élargi à de trop nombreux acteurs et hétérogène sur le plan des problématiques sociales et sanitaires » mais aussi celles des élus du conseil départemental souhaitant conserver l'héritage de la construction «par le bas » des politiques médicosociales avec les anciennes DDASS [10].

9 Neufs signataires : la préfecture du département, le conseil départemental, deux communes (bourgs-centre des deux cantons rassemblés en un Pays), la Caisse d'allocations familiales (CAF), la Mutualité sociale agricole (MSA), la Caisse primaire d'assurance maladie (CPAM), la Direction des services départementaux de l'éducation nationale (DDSEN) et l'ARS.
} 
2009, et sur lequel l'ARS refuse de s'engager financièrement.

Au-delà de ces indicateurs factuels, l'ARS, dont le contrat d'objectifs et de moyens prévoit la signature de deux CLS avant 2013 et qui a pris l'initiative de choisir ce «territoire rural», a dû mobiliser et convaincre les collectivités de s'engager dans la démarche: leur accord s'est scellé au prix d'un arrangement - négocié de façon informelle avec les élus de ne pas fermer ce plateau chirurgical local. «En échange», la «jeune» ARS soucieuse d'affirmer son leadership territorial, demande aux élus de retirer le volet santé d'un Contrat de revitalisation signé entre plusieurs communes et les conseils départemental et régional. Elle propose que ce volet fasse l'objet d'un CLS.

En charge d'animer la fabrication du CLS en «seulement deux mois et six réunions $d u$ comité opérationnel », les agents de la DT de l'ARS ont alors mobilisé les acteurs locaux ce qui, selon leurs propos, a conduit à leur «engagement tous azimuts... mais qui finalement, est parti dans tous les sens et on n'a plus rien maîtrisé!». De plus, la gouvernance de la démarche contractuelle s'est révélée complexe car organisée autour de trois instances aux prérogatives différenciées et peu intégrées: un comité de pilotage «stratégique et politique», composé en majorité d'élus locaux ${ }^{10}$, chargé de valider les axes et contenus du CLS ; un comité opérationnel «plus technique» rassemblant professionnels de santé, techniciens territoriaux, associations, réseaux de santé et entreprises, chargé de fabriquer des fiches-actions; et un comité de rédaction restreint à six représentants des signataires «politiques» (conseil départemental, bourgs-centre et DT) chargé de «lisser les conflits» et de finaliser le document contractuel pour sa signature. Par exemple, le comité opérationnel est «composé officiellement» et sur décision du comité de pilotage, de 32 membres. Mais en réalité, 50 à 60 personnes différentes participent à ses travaux entre mars et mai 2011. D'une part parce qu'elles sont déjà engagées dans des dispositifs médicosociaux; d'autre part, pour faire valoir leurs compétences et tenter d'assurer, via le CLS, la pérennité de leur emploi ou de leurs subventions selon une logique de «guichet». Ainsi, les réunions sont ponctuées par des interrogations des participants sur le sens et l' «intérêt à s'engager dans ce contrat», dans un environnement qu'ils jugent «incertain et qui bouge en permanence» en l'absence d'un PSRS validé; elles sont aussi un lieu de concurrence entre opérateurs publics et privés trouvant dans le CLS une opportunité de faire reconnaître et financer leurs actions en contexte de restriction budgétaire. Ces interrogations et concurrences ont non seulement complexifié et ralenti le travail de négociations mais aussi favorisé une surenchère de propositions. Les travaux de ce comité débouchent, selon l'ARS, sur une «inflation» de fiches-actions (31 au lieu des 12 prévues initialement): 20 sont sélectionnées par le comité de pilotage en intégrant, malgré des protestations virulentes des membres du comité opérationnel, des fiches «venues d'en haut et imposées » par l'ARS, le conseil départemental et des opérateurs régionaux. Suite à un «boycott» du dernier comité de pilotage par les élus locaux estimant «ne pas avoir été tenus suffisamment informés » et l'arrêt des négociations pendant six mois, celles-ci reprennent et aboutissent à un accord sur le choix des trois axes stratégiques du CLS, en

\footnotetext{
${ }^{10}$ Le comité de pilotage est composé de 9 personnes : 5 élus locaux, le délégué territorial de l'ARS, le secrétaire général de la préfecture et deux représentants de l'Assurance maladie.
} 
janvier 2012, soit 15 mois après le lancement de la démarche. L'écriture du contrat par un comité de rédaction volontairement réduit, permettra, au prix de quelques arrangements et «bricolages» entre parties prenantes, d'assurer la signature officielle du premier CLS de la région en juillet 2013 [22].

\subsection{Cas B. Mobiliser des partenaires et ouvrir un dialogue social territorial : une gouvernance rapprochée}

Dans ce cas, la même démarche ne durera «seulement que 15 mois» (octobre 2012 février 2014), selon un rythme de travail intense mais continu et sans désaccords majeurs entre «partenaires». A l'inverse du cas A, c'est une commune et non l'ARS qui initie la démarche de contractualisation: portée par un fort volontarisme politique du maire, elle est consolidée par la mobilisation préalable de son directeur général des services, de quatre directions municipales ${ }^{11}$ mais aussi la création d'un Comité consultatif d'accès aux soins permettant d'intégrer les professionnels libéraux de santé aux négociations et de prendre en compte leurs avis et attentes.

Si le diagnostic initial quantitatif est réalisé de façon identique à celui du cas $\mathrm{A}$, il est complété par un diagnostic «qualitatif et partagé», mené par l'ARS qui recrute à cet effet une stagiaire. Construit sur la base d'entretiens et de questionnaires auprès des institutions et professionnels locaux des secteurs médical, social et médicosocial, ce travail débouche sur un diagnostic sanitaire territorialisé qui fait consensus entre l'ARS, les élus et services municipaux, les établissements, professionnels et associations de santé œuvrant sur la commune. Lors de l'unique réunion du comité de pilotage, qualifié de «simple chambre d'enregistrement» des propositions du comité opérationnel, ce double diagnostic aboutit rapidement à un accord sur les axes du CLS à privilégier. De ce fait, des directives précises de réflexion sont données au comité opérationnel organisé en groupes de travail spécialisés sur chacun des axes. Cette rapidité est aussi facilitée par le fait que la commune est seule signataire du CLS avec l'ARS et qu'elle a embauché, «à ses frais », une chargée de mission pour préparer, animer, orienter les débats des groupes de travail et réaliser des comptes-rendus soumis à l'approbation du comité opérationnel. Le pilotage des réunions par cette chargée de mission est assuré en étroite collaboration avec le directeur du Centre communal d'action sociale et le directeur général adjoint des services qui assurent le suivi du contrat et en rendent régulièrement compte au maire-adjoint délégué à la santé et à l'ARS.

Ce portage quasi exclusivement «municipal», accepté par l'ARS faute de moyens humains disponibles pour le suivi, a facilité une rédaction localement «négociée » du contrat avec les professionnels de santé et associations locales. Il a surtout pris appui sur une démarche de projet interservices inscrite de longue date dans les «habitudes de travail en commun» entre services municipaux et avec les partenaires locaux. Cette gouvernance rapprochée a permis aux élus et techniciens territoriaux de cadrer les négociations lors des groupes de travail, de sélectionner les «bons interlocuteurs» locaux «qui travaillaient déjà sur les axes et que l'on connaissait bien et depuis longtemps » et de rédiger, en moins de deux mois, le CLS et 10 fiches-actions

${ }^{11}$ Cohésion sociale, Centre communal d'action sociale, Enfance-famille-éducation-sports, Restauration collective. 
plutôt de type «fiche-orientation». Comme le précise un élu, il s'agissait plutôt, compte tenu de la proximité des élections municipales de mars 2014, d' "une feuille de route générale pour la prochaine mandature».

Le suivi de la fabrication du contrat par une «équipe-projet volontairement restreinte » et une connaissance affinée du terrain ont donc joué un rôle central dans la construction et l'acceptation rapide du CLS «en l'état»par la direction de l'ARS. Appréciant la mise en relation de ses axes prioritaires avec ceux du PSRS et la dynamique locale et partenariale engendrée autour de la réduction des inégalités sociales de santé, l'ARS n'a que peu modifié les propositions. Ses agents «... ont juste corrigé quelques virgules » diront les techniciens communaux et les élus ont «évité de parler des financements» du CLS, remettant cette négociation «qui risquait de fâcher l'ARS» à plus tard, lors de la mise en œuvre du contrat.

In fine, les débats et controverses qui traversent la gouvernance négociée des CLS entre ARS et collectivités territoriales, traduisent une tension entre deux registres d'action et d'interactions: un CLS conçu souvent a priori par l'ARS selon une logique top down et le considérant comme une «simple» déclinaison locale du PSRS à laquelle ont fortement résisté les «partenaires » locaux (cas A); un CLS à dimension plus participative et de type bottom-up, porteur de solutions ajustées au plus près des problématiques sanitaires du territoire et de ses populations. Encastré dans des politiques transversales de par l'engagement des élus et directeurs de services municipaux, il est conçu dans ce cas (B), comme un instrument de «démocratie sanitaire » et de coordination entre acteurs locaux de santé. La comparaison des formes de gouvernance de ces deux CLS, montre aussi qu'un processus d'apprentissage du partenariat traverse leur fabrication. Suite au changement de la direction générale de l'ARS en 2012, celle-ci semble avoir tiré des enseignements du premier CLS signé (cas A) : tenue d'adopter un mode de gouvernance plus participatif et mieux instrumenté pour le second, elle reconnaît toutefois avoir aussi, dans ce cas, "perdu la main... comme un aveu que l'on a besoin que les collectivités soient présentes... ce qui démontre notre impuissance».

Les formes différenciées de gouvernance des CLS étudiés montrent que cette procédure contractuelle au caractère peu formalisé $a$ priori est relativement instable dans le temps et l'espace, cas de figure que l'on retrouve dans des programmes nationaux déclinés régionalement [8]. Elle comporte une grande part d'indétermination quant à sa territorialisation, sa trajectoire et son aboutissement: signer un contrat négocié assurant l'articulation entre politiques régionale et locales de santé et censé lutter contre les cloisonnements intersectoriels du système sanitaire et les inégalités sociales et territoriales de santé. Cette politique contractuelle «des petits pas» tient en partie à son «flou » initial que l'on peut considérer comme une ressource autorisant des arrangements diversifiés selon les configurations locales et sectorielles. Il constitue aussi un frein à la construction des accords lorsque le mode de gouvernance territoriale adopté par l'ARS est ressenti par les acteurs locaux comme trop (re)centralisé d'autant plus quand il se double d'incertitudes quant au financement et à la pérennisation des actions. Les effets propres des territoires, entendus non seulement dans leur dimension géographique, mais aussi comme constructions sociales, historiques, économiques et politiques, s'ils sont ignorés ou négligés par l'ARS, constituent aussi des nœuds de résistance aux négociations. Enfin, pour les élus et professionnels locaux 
de santé, le CLS apparaît alternativement comme une opportunité pour faire valoir une légitimité politique, mobiliser des expertises et réseaux professionnels, pérenniser des emplois et améliorer l'attractivité des structures hospitalières locales. Toutefois, il représente aussi un risque de mise en concurrence accrue des opérateurs de proximité au nom des principes d'efficience, de réduction des coûts et de mutualisation des moyens. Loin d'être un instrument d'unification des démarches contractuelles, comme proposé par le «kit méthodologique» national, un CLS s'apparente davantage à une obligation de fabriquer du «sur mesure» répondant aux spécificités des territoires infrarégionaux tout en prenant en compte les orientations du PSRS.

\section{CONCLUSION}

Malgré ces figures protéiformes de gouvernance, les CLS se négocient bien ou mal, en douceur ou dans une grande pénibilité, une partie des problèmes finissant par trouver des solutions [9]. Alors que les ajustements se font parfois difficilement tant les luttes de pouvoir marquent les échanges et débouchent sur des adaptations bricolées, le jeu d'instrumentalisations réciproques [13] apparaît comme une condition majeure facilitant une gouvernance négociée entre acteurs placés en situation à la fois de coopération obligée et de concurrence [23]. En termes de recommandations, plusieurs conditions semblent favorables à la construction des accords. Un portage politique par les élus locaux, la réalisation d'un double diagnostic quantitatif et qualitatif partagé et à base consultative afin de définir des objectifs stratégiques en amont des groupes de travail et mobiliser les futurs «partenaires » locaux, un nombre restreint de signataires mais une intense activité de coordination des ressources de proximité - acteurs professionnels et associatifs œuvrant dans différents secteurs d'intervention sur le territoire et au-delà dans les politiques publiques locales - et une participation des habitants et représentants d'usagers. Enfin, la maîtrise d'une ingénierie de projet territorial, outre sa fonction d'instrument de gouvernance, permet de concrétiser les objectifs en programmes d'actions à mettre en œuvre et d'identifier précisément les contributions conjointes de chacun des «partenaires parties prenantes » engagés dans un CLS.

Les limites de cette recherche tiennent en partie aux exigences temporelles d'une méthodologie socio-ethnographique nécessitant de suivre au long cours la fabrication de nombreux CLS pour apprécier, au plus près des acteurs, les processus d'action publique à l'œuvre dans ces politiques contractuelles. Le choix de procéder ici par effets de contraste entre deux cas distincts, s'il permet de baliser le spectre des variations de la gouvernance territoriale des CLS et d'en comprendre les ressorts, mérite d'être étendu à d'autres CLS pour saisir des modes de gouvernance plus nuancés et composites. Enfin, les effets de la réforme territoriale actuelle et plus particulièrement la fusion des régions et le développement des métropoles, viennent rajouter de l'incertitude et interrogent les modes de gouvernance sanitaire des «nouveaux » périmètres et territoires de santé qui seront adoptés ${ }^{12}$.

\footnotetext{
${ }^{12}$ Cette recherche a été financée par l'Agence nationale de la recherche (2012-2015) et a bénéficié d'un soutien financier de l'ARS (2013-2014) ainsi que de la Ligue nationale contre le cancer.
} 


\section{BIBLIOGRAPHIE}

[1] Le Galès P. Régulation, gouvernance et territoire. Dans: Les métamorphoses de la régulation politique. Paris: LGDJ. 1998: 203-240.

[2] Jabot F, Demeulemeester R. Les interactions entre les niveaux national, régional et infrarégional dans les programmes de santé publique. Santé Publique 2005; 17 (4): 597-606.

[3] Duran P, Thoenig JC. L'Etat et la gestion publique territoriale. Revue française de science politique I996; 46 (4): 580-663.

[4] Pasquier R, Simoulin V, Weisbein J (dir.). La "gouvernance territoriale»: théories, pratiques et discours. Paris: LGDG. 2007, 235 p.

[5] Halpern C, Lascoumes P, Le Galès P (dir.). L'instrumentation de l'action publique. Controverses, résistances, effets. Paris: Presses de Sciences Po. 2014, 520 p.

[6] Gaudin JP. Gouverner par contrat. L'action publique en questions. Paris: Presses de Sciences Po. 1999, 275 p.

[7] Nay O, Smith A. Les intermédiaires en politique. Médiations et jeux d'institutions. Dans: Le gouvernement des compromis. Courtiers et généralistes dans l'action publique. Paris: Economica. 2002: 1-21.

[8] Honta M, Haschar-Noé N, Sallé L. La fabrique des territoires de santé publique en France. Une analyse comparée de la mise en œuvre du Programme national nutrition santé en Aquitaine, Midi-Pyrénées et Nord-Pas de Calais. Cahiers de Géographie du Québec 2011; 55 (156): 379-397.

[9] Lorrain D. Gouverner «dur-mou » : neuf très grandes métropoles. Revue française d'administration publique, 2003 ; 3 (107): 447-454.

[10] Hudebine H, Jourdain A, Munoz J. Les premières années des ARS dans le secteur médicosocial. Journal de gestion et d'économie médicales 2014; 31 : 81-96.

[11] Pierru F. La santé en fusions. L'accouchement des agences régionales de santé au forceps institutionnel. Dans: La territorialisation des politiques de santé. Bordeaux: Editions Hospitalières. 2012:77-108.
[12] Smith A, Sorbets C. Le leadership politique et les territoires. Les cadres d'analyse en débat. Rennes: Presses universitaires de Rennes. 2003, 293 p.

[13] Lacuisse ME, Mayaux PL. L'action publique et la multiplication des niveaux de gouvernement. Dans: Quand les politiques changent. Temporalités et niveaux de l'action publique. Paris: L'Harmattan. 2010: 215-245.

[14] Dubois V.Ethnographier l'action publique. Les transformations de l'Etat au prisme de l'enquête de terrain. Gouvernement et action publique 2012; 1: 83-101.

[15] Jouve B, Lefèvre C. De la gouvernance urbaine au gouvernement des villes? Permanence ou recomposition des cadres de l'action publique en Europe. Revue française de science politique 1999;49 (6) : 835-854.

[16] Offner JM. Les territoires de l'action publique locale. Fausses pertinences et jeux d'écarts. Revue française de science politique 2006; 56 (1): 27-47.

[17] Raulet-Croset N. La dimension territoriale des situations de gestion. Revue française de gestion 2008; 184: 137-150.

[18] Jamet P. Une nouvelle gouvernance territoriale des politiques sociales est-elle possible? Pouvoirs Locaux 2015; 105: 51-54.

[19] Le Lidec P. Le jeu du compromis : l'Etat et les collectivités territoriales dans la décentralisation en France. Revue française d'administration publique 2007; 1 (121-122): 111-130.

[20] Clavier C. Les élus locaux et la santé: des enjeux politiques territoriaux. Sciences sociales et santé 2009; 27: 47-74.

[21] Garnier J. Appareil productif, territoire, dialogue social: pourquoi innover? Innovations $2012 ; 38$ : 164-184.

[22] Haschar-Noé N, Salaméro E. La fabrication d'un contrat local de santé «expérimental»: négociations et compromis sous tensions. Sciences sociales et santé 2016 ; ; 34 (2): 81-105.

[23] Mabileau A. Les génies invisibles du local. Faux-semblants et dynamiques de la décentralisation. Revue française de science politique 1997; 3 (47) : 340-376. 